\title{
Efectividad del tratamiento con bortezomib en pacientes con mieloma múltiple en el Hospital de Clínicas en el período 2009-2016. Montevideo, Uruguay
}

Sabrina Ranero*, Virginia Bove†, Fiorella Villanoł, Lilián Díazs, Eloísa Riva

\section{Resumen}

El mieloma múltiple representa la segunda neoplasia hematológica en frecuencia. Es una enfermedad incurable, cuya sobrevida se ha duplicado en los últimos años, vinculado esto a la aparición de nuevos fármacos. Los planes con bortezomib mejoran la respuesta, sobrevida libre de progresión y sobrevida global. En Uruguay, desde 2009, este fármaco tiene cobertura por parte del Fondo Nacional de Recursos. Este estudio tiene como objetivo analizar la efectividad y toxicidad de bortezomib en pacientes con diagnóstico de mieloma múltiple asistidos en el Hospital de Clínicas.

Método: estudio observacional, retrospectivo y descriptivo en el que se incluyeron todos los pacientes con diagnóstico de mieloma múltiple que recibieron tratamiento con bortezomib en primera, segunda o tercera línea en el Hospital de Clínicas de Montevideo, Uruguay, desde 2009 a 2016.

Resultados: 36 pacientes recibieron bortezomib. El plan más utilizado fue ciclofosfamida-bortezomib-dexametasona. La vía de administración fue subcutánea en $53,8 \%$. La tasa de respuesta global fue de $79,5 \%$ ( $87 \%$ en primera línea y $68,8 \%$ en segunda o tercera línea). El 47,2\% desarrolló polineuropatía y 30,6\% citopenias. Con una mediana de seguimiento de 26 meses la sobrevida global fue de $61 \%$ y la sobrevida libre de progresión de 35 meses (IC 95\%, 22,6-47,4).

Conclusión: el tratamiento con bortezomib logró buena tasa de respuesta. La neuropatía fue la toxicidad más frecuente. Bortezomib es un fármaco efectivo y con adecuado perfil de seguridad para el tratamiento del mieloma múltiple en primera, segunda y tercera línea.

Palabras clave: Mieloma múltiple

Bortezomib

Resultado del tratamiento

Key words: Multiple myeloma

Bortezomib

Treatment outcome

\footnotetext{
* Residente de Hematología, Hospital de Clínicas. Montevideo, Uruguay.

† Hematóloga, Hospital de Clínicas. Montevideo, Uruguay.

¥ Hematóloga, Hospital de Clínicas. Montevideo, Uruguay.

$\S$ Profesor Titular de Hematología, Hospital de Clínicas. Montevideo, Uruguay.

II Profesor Adjunto de Hematología, Hospital de Clínicas. Montevideo, Uruguay.

Correspondencia: Dra. Eloísa Riva. Hospital de ClínicasDr. Manuel Quintela. Av. Italia s/n. Montevideo, Uruguay.

Correo electrónico: eloisariva@hotmail.com

No presentamos conflicto de intereses.

Trabajo aprobado por el Comité de Ética del Hospital de Clínicas

Recibido: 23/1/18

Aprobado: 15/8/18
} 


\section{Introducción}

El mieloma múltiple (MM) representa la segunda neoplasia hematológica en frecuencia, con una incidencia de 4-6/100.000/año y una mediana de edad al diagnóstico de 69 años. Se caracteriza por la presencia de una proteína monoclonal que determina daño orgánico incluyendo lesiones líticas óseas, anemia, falla renal e hipercalcemia ${ }^{(1)}$.

Es una enfermedad incurable cuya sobrevida se ha duplicado en los últimos años, vinculado esto a la aparición de nuevos fármacos (bortezomib, lenalidomida, talidomida) y a la utilización del trasplante de progenitores hematopoyéticos (TPH), alcanzando actualmente 6-7 años. La estrategia terapéutica actual, tanto en candidatos como en no candidatos a TPH, incluye regímenes con tres fármacos. Múltiples ensayos clínicos han demostrado que la combinación de inhibidores de proteosomas con corticoides, alquilantes o inmunomoduladores mejoran significativamente las tasas respuesta y control de la enfermedad ${ }^{(2)}$.

Bortezomib es un inhibidor selectivo y reversible del proteosoma 26S, que controla una ruta clave en la proliferación celular mediada por el factor nuclear $\kappa \beta$ $(\mathrm{NF} \kappa \beta)$ y su inhibidor. La inhibición del proteosoma 26S bloquea la activación del NFк $\beta$, provocando una disminución de las señales de proliferación celular y favoreciendo la apoptosis. Inhibe, además, a los efectores que intervienen en la reparación del ácido desoxirribonucleico $(\mathrm{ADN})$ e interfiere en las interacciones entre células plasmáticas y el microambiente ${ }^{(3)}$.

Fue aprobado para su uso en MM en 2003, basado en el ensayo SUMMIT multicéntrico, fase II, que mostró respuesta global en 35\% de los pacientes con MM refractario o recaído a tratamientos previos ${ }^{(2)}$.

Posteriormente, se observó ventaja en la sobrevida global (SG) con su asociación a inmunomoduladores para el tratamiento del MM en primera línea ${ }^{(4)}$.

En Uruguay, el uso de bortezomib en MM se aprobó con cobertura por parte del Fondo Nacional de Recursos (FNR) para MM de alto riesgo al debut (falla renal o citogenética adversa) y ante recaída de la enfermedad en el período 2009-2018, ampliándose recientemente dicha normativa.

Este estudio tiene como objetivo analizar la efectividad y toxicidad de bortezomib en pacientes con diagnóstico de MM en el período enero de 2009 a diciembre de 2016 en la Unidad de Mieloma y Amiloidosis de la Cátedra de Hematología del Hospital de Clínicas.

Este es el primer reporte de datos nacionales referido a resultados obtenidos, en términos de respuesta y toxicidad, en pacientes con MM tratados con bortezomib en nuestro país.

\section{Materiales y método}

\section{Fuentes de datos}

Se realizó un estudio observacional, retrospectivo y descriptivo en el que se incluyeron todos los pacientes con diagnóstico de MM que recibieron tratamiento con bortezomib en primera, segunda o tercera línea en el Hospital de Clínicas de Montevideo, Uruguay, en el período enero de 2009 a diciembre de 2016 asistidos en la policlínica de gamapatías monoclonales de dicho hospital. Se excluyeron aquellos pacientes tratados con bortezomib más allá de la tercera línea de tratamiento o en contexto de mantenimiento.

\section{Eventos clínicos analizados}

Se registraron datos patronímicos, características clínicas y de laboratorio de la enfermedad, plan de tratamiento, vía de administración de bortezomib (intravenosa o subcutánea), respuesta al tratamiento y toxicidad del mismo, modificaciones del plan terapéutico, realización de trasplante autólogo de progenitores hematopoyéticos (auto-TPH), requerimiento de hemodiálisis (HD) y mortalidad.

Se confeccionó una planilla de recolección de datos ad hoc completada prospectivamente.

El diagnóstico de MM se basó en los criterios diagnósticos del Grupo de Trabajo Internacional de Mieloma $(\mathrm{IMWG})^{(5)}$.

La respuesta al tratamiento fue clasificada en cinco grupos siguiendo los criterios del IMWG que consideran las siguientes categorías: remisión completa (RC); muy buena remisión parcial (VGPR); remisión parcial (PR); enfermedad estable (EE), y progresión ${ }^{(6)}$.

La respuesta global (RG) en nuestro estudio comprende la suma de RC, VGPR y PR.

Se consideró MM de alto-intermedio riesgo citogenético la presencia por hibridación in situ fluorescente (FISH) de deleción $17 \mathrm{p}, \mathrm{t}(14 ; 16)$ o t $(4 ; 14)$, y por citogenética convencional la deleción 13q, hipoploidía o amplificaciones 1q. No se realizó selección de células plasmáticas previo a la evaluación citogenética por no estar disponible la técnica en ese período en nuestro país.

Consideramos toxicidad relacionada al tratamiento la aparición de al menos una de las siguientes: neuropatía periférica (NP) de cualquier grado; una o más citopenias grado 3-4, según criterios del National Cancer Institute Common Toxicity Criteria NCI; herpes zóster ${ }^{(7)}$.

Bortezomib se utilizó de inicio a dosis estándar de $1,3 \mathrm{mg} / \mathrm{m}^{2}$ semanal los días $1,8,15$ y 21 de cada ciclo de quimioterapia. La vía de administración fue intravenosa hasta setiembre de 2013, siendo posteriormente reemplazada por la vía subcutánea. En caso de aparición de NP los ajustes de dosis de bortezomib fueron los si- 
guientes: si NP grado 1-2, disminuir bortezomib a 1 $\mathrm{mg} / \mathrm{m}^{2}$; si NP grado 2 dolorosa o grado 3 , suspender bortezomib hasta resolución y reinstalar a dosis de 0,7 $\mathrm{mg} / \mathrm{m}^{2}$; si NP grado 4 , suspensión definitiva.

La supervivencia libre de progresión (SLP) se define como el tiempo desde la fecha de inicio de tratamiento con bortezomib hasta la fecha de progresión de la enfermedad o muerte, o último contacto si el paciente se encontraba en remisión.

La SG hace referencia al tiempo entre el inicio de tratamiento hasta la fecha de muerte o fecha del último contacto si el paciente permanecía vivo.

\section{Análisis estadístico}

Para el análisis estadístico se utilizó la base de datos SPSS (Statistical Package for the Social Science, versión 22.0). A todas las variables se les realizó la determinación de frecuencia; en las variables continuas se determinó medidas de resumen (mediana y media). Las curvas de sobrevida fueron construidas según el método de Kaplan-Meyer y para su comparación se utilizó el test log-rank. En todos los casos se tomó un valor de $\mathrm{p}$ $<0,05$ como significativo.

\section{Resultados}

Durante el período de estudio, 36 pacientes con diagnóstico de MM recibieron tratamiento con bortezomib en nuestra institución. Tres de ellos lo recibieron en dos oportunidades. La distribución de los pacientes según sexo fue igual, 18 hombres y 18 mujeres, con una mediana de edad de 57 años (rango 37-77 años), siendo $63,9 \%(\mathrm{n}=23)$ de los pacientes $\leq 65$ años. Las características de los pacientes se describen en la tabla 1 .

Al inicio del tratamiento, $69,4 \%$ tenía lesiones líticas; $58,3 \%$ anemia (hemoglobina $<10 \mathrm{~g} / \mathrm{dl}$ ); $44,4 \%$ insuficiencia renal (creatinina $\geq 2 \mathrm{mg} / \mathrm{dl}$ ), y $19,4 \%$ hipercalcemia (calcio total $\geq 11 \mathrm{mg} / \mathrm{dl}$ ). Diez pacientes $(27,8 \%)$ requirieron $\mathrm{HD}$ al debut, quedando seis en HD crónica $(16,7 \%)$.

La distribución de los pacientes de acuerdo al subtipo de Ig fue: $\operatorname{IgG} 50 \%(\mathrm{n}=18)$; $\operatorname{IgA} 30,6 \%(\mathrm{n}=11)$; cadenas livianas $16,7 \%(\mathrm{n}=6)$; $\operatorname{IgM} 2,8 \%(\mathrm{n}=1)$.

La mayoría presentaba un estadio avanzado de la enfermedad: $86,1 \%(\mathrm{n}=31)$ estadio III de Durie y Salmon y $77,8 \%(\mathrm{n}=28)$ ISS $\geq 2$.

Los estudios citogenético convencional y FISH se realizaron en $35 / 36$ pacientes, no se encontraron alteraciones en $28(80 \%)$ y $26(74,3 \%)$ casos, respectivamente. Se documentaron alteraciones de alto riesgo en seis casos por citogenética convencional (hipoploidía: 3, del13q: 2, amplificación 1q:1) y en cinco casos en el FISH (del17p: 2, t[14;16]: 2 y t[4;14]: 1).
Tabla 1. Características de los pacientes $(n=36)$.

Performance estatus según ECOG

$0-2$

$88,6 \%$

3-4

$11,4 \%$

Estadio Durie y Salmon

II

$13,9 \%$

III

$86,1 \%$

ISS

$122,2 \%$

$2 \quad 30,6 \%$

$3 \quad 47,2 \%$

Albuminemia $<3,5 \mathrm{~g} / \mathrm{dl} \quad 41,7 \%$

$\beta_{2}$-microglobulina $\geq 5,5 \mathrm{mg} / \mathrm{dl} \quad 47,2 \%$

$\mathrm{Hb}<8,5 \mathrm{~g} / \mathrm{dl} \quad 38,9 \%$

LDH elevada (disponible en 19) $\quad 57,9 \%$

VES $>100$ (disponible en 18) $\quad 33,3 \%$

*ECOG: Eastern Cooperative Oncology Group; LDH: lactato deshidrogenasa; VES: velocidad de eritrosedimentación.

Se indicó bortezomib a la dosis convencional de 1,3 $\mathrm{mg} / \mathrm{m}^{2}$ semanal en todos los casos; la vía de administración fue subcutánea en 53,8\% $(\mathrm{n}=21)$, los casos restantes lo recibieron intravenoso. La mediana de ciclos recibidos fue de 6 (rango 1-9).

Bortezomib fue utilizado en primera línea en 59\% $(n=23)$, en segunda línea en 30,8\% ( $n=12)$ y en tercera línea en $10 \%(\mathrm{n}=4)$. Los planes de tratamiento se describen en la figura $1 \mathrm{~A}$, siendo el más frecuentemente utilizado el triple plan ciclofosfamida, bortezomib y dexametasona (CyBorD), tanto en candidatos como en no candidatos a TPH.

La RG fue 79,5\% (n=31); 20,5\% (n=8) RC, y 28,2\% $(n=11)$ VGPR. Seis $(15,4 \%)$ pacientes fueron refractarios al tratamiento (cuatro EE y dos progresión). En dos pacientes la respuesta no pudo ser valorada por fallecimiento precoz. Diecisiete $(43,6 \%)$ pacientes recibieron consolidación con TPH, siete de ellos lograron mejorar la respuesta al tratamiento luego del mismo. La RG fue de $87 \%(20 / 23)$ y $68,8 \%(11 / 16)$ en los que recibieron bortezomib en primera línea y en segunda o tercera línea, respectivamente. En cuanto a la profundidad de la respuesta, los pacientes que recibieron bortezomib en primera línea lograron RC 21,1\% y VGPR 39,1\%, en tanto los que lo recibieron en segunda o tercera línea obtuvieron RC 18,8\% y VGPR 12,5\% (figura 1B). 


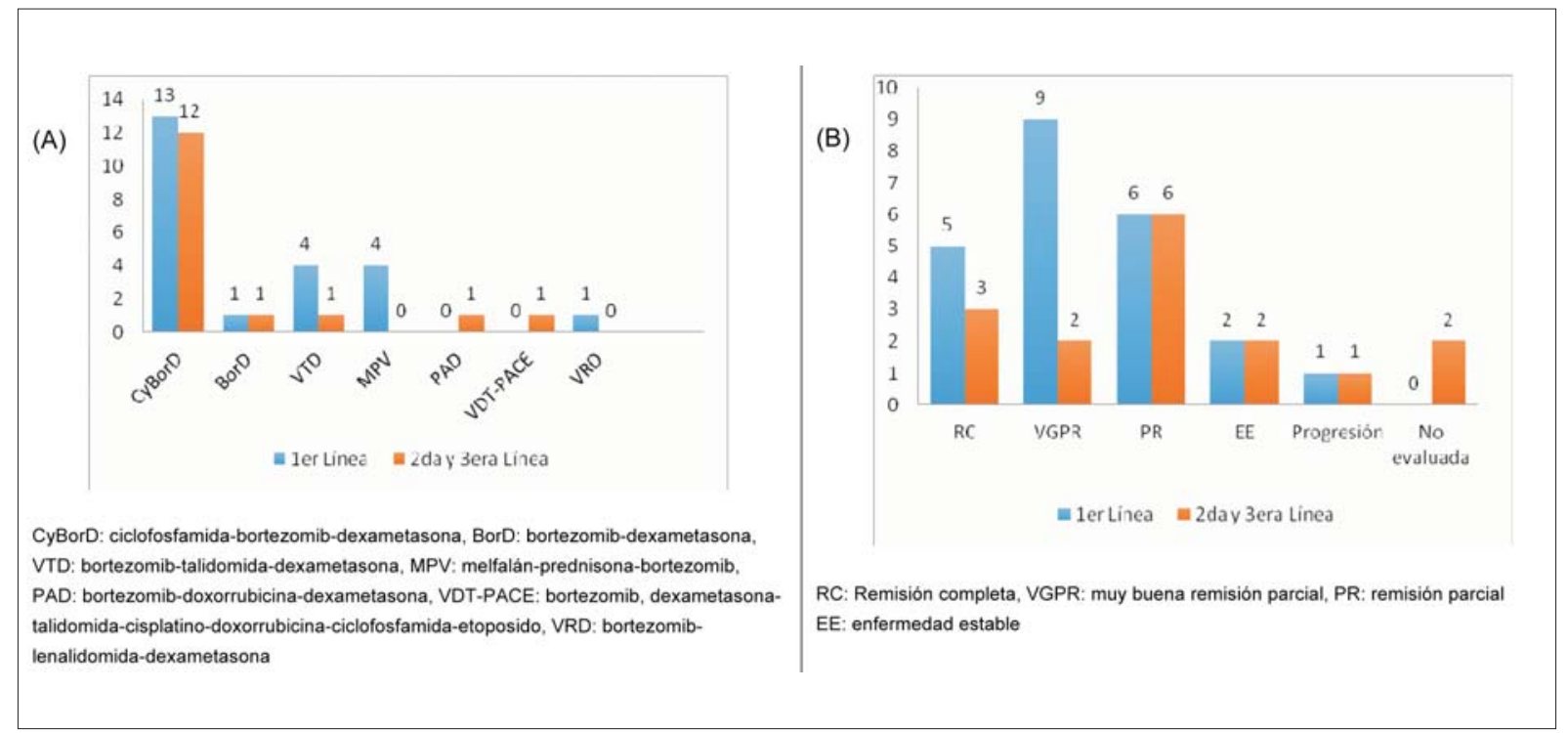

Figura 1. (A) Planes de tratamiento en $1^{\mathrm{a}}$ y $2^{\mathrm{a}}-3^{\mathrm{a}}$ línea. (B) Respuesta al tratamiento en $1^{\mathrm{a}}$ y $2^{\mathrm{a}}-3^{\mathrm{a}}$ línea.

Más de la mitad de los casos $(61 \%, \mathrm{n}=22)$ presentó al menos una toxicidad al tratamiento (tabla 2), en ningún caso fatal. En 17 pacientes (47,2\%) hubo neurotoxicidad de cualquier grado, $36,1 \%(\mathrm{n}=13) \mathrm{NP}$ grado $1 \mathrm{y}$ $8,3 \%(\mathrm{n}=3)$ NP grado 2 y $1(2,8 \%)$ NP grado 3 ; este último paciente recibía un plan que asociaba dos fármacos neurotóxicos: bortezomib y talidomida (VTD). Desarrolaron NP de algún grado 55,6\% (10/18) de los que recibieron bortezomib intravenoso y 33\% (7/21) de los subcutáneos. La RG fue de 88,9\% (RC 11,1\%, VGPR $27,8 \%$ ) y $71,5 \%$ (RC $28,6 \%$, VGPR $28,6 \%$ ) para los pacientes que lo recibieron intravenoso y subcutáneo, respectivamente.

La toxicidad hematológica se documentó en 30,6\% $(n=11)$, siendo grado 2-3 en nueve pacientes (dos plaquetopenia, tres anemia y cuatro neutropenia) y grado 4 en dos casos.

Todos recibieron profilaxis con aciclovir, dos presentaron herpes zóster cutáneo.

La dosis de bortezomib se redujo en cuatro pacientes, en todos los casos por NP. En cuatro pacientes el tratamiento fue suspendido; dos por infección, uno por fallecimiento precoz, uno por abandono de tratamiento.

La SG a 26 meses fue de 61\% (figura $2 \mathrm{~A}$ ).

Globalmente, la mediana de SLP fue de 35 meses (IC $95 \%, 22,647,4)$ (figura 2B). La mediana de SLP para los que recibieron bortezomib en primera línea fue de 43 meses (IC 95\%, 12,173,9) y 29 meses en segunda o tercera línea (IC 95\%, 22,835,2; $\mathrm{p}=0,883$ ).

Al momento del análisis la mortalidad fue de $47,2 \%$ (17/36) y las causas de fallecimiento fueron infección $(n=6)$, progresión $(n=9)$, infarto agudo de miocardio $(\mathrm{n}=1)$. En un caso no se encontró dato de causa de muerte.

\section{Discusión}

El diagnóstico y tratamiento del MM ha experimentado avances trascendentes en los últimos años. Los criterios de inicio de tratamiento actuales permiten adelantarnos al desarrollo de daño de órgano blanco ${ }^{(1)}$. Estos criterios no habían sido implementados en el período analizado, por lo cual todos los pacientes incluidos en el presente estudio tenían, por lo menos, daño de un órgano blanco.

Los pacientes incluidos en este estudio recibieron bortezomib con cobertura del FNR en todos los casos. En función de los criterios aceptados, constituye una población de alto riesgo, con estadio avanzado (86\%) y score ISS 2-3 en 78\%.

Ls SG estimada para esta población es inferior a tres años ${ }^{(8-10)}$. Los planes de tratamiento con nuevas drogas, particularemente inhibidores de proteosomas e inmunomoduladores, y más recientemente anticuerpos monoclonales, han permitido mejorar SLP y SG. Los planes triples con bortezomib han mostrado mayor SLP y SG que planes basados en inmunomoduladores y dexametasona ${ }^{(11)}$; asimismo, el uso de bortezomib-talidomida-dexametasona obtiene mayor profundidad de respuesta y SG comparado con planes de dos fármacos ${ }^{(12)}$.

Los planes basados en bortezomib han demostrado superar el pronóstico adverso de la $\mathrm{t}(4 ; 14)$ y parcialmente la del17p, tanto en candidatos como en no candidatos a $\mathrm{TPH}^{(13)}$.

En función de la evidencia científica, las pautas internacionales y la disponibilidad de fármacos en nuestro 


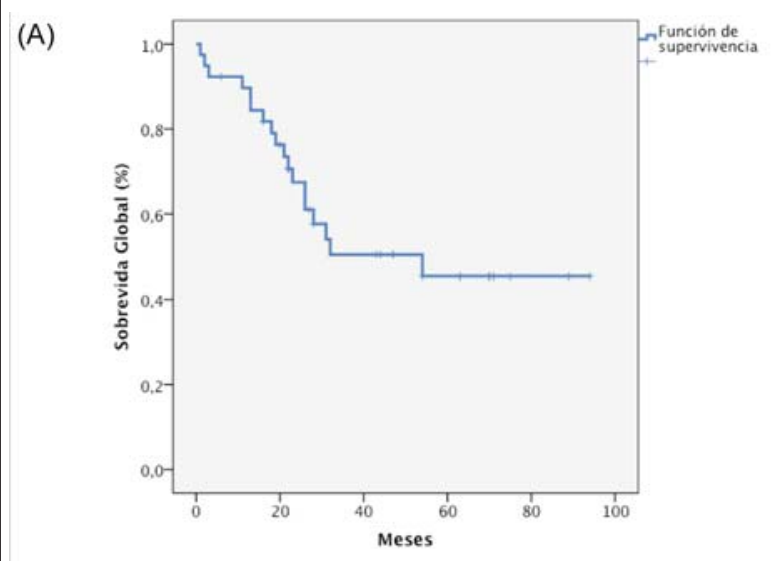

(B)

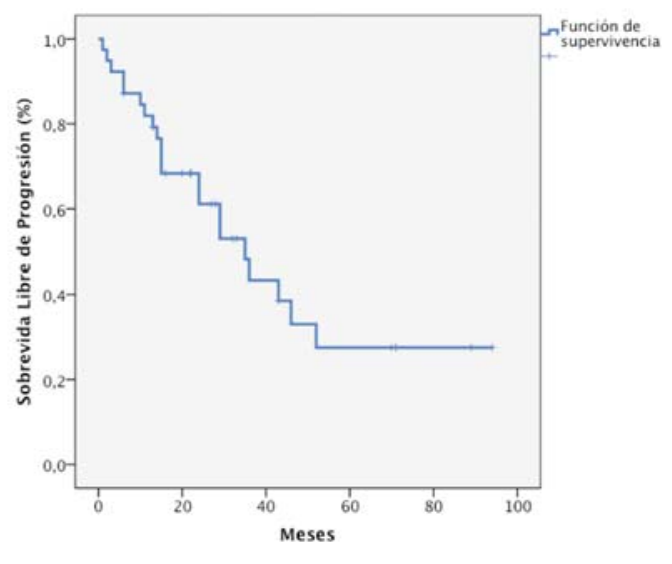

Figura 2. (A) Sobrevida global. (B) Sobrevida libre de progresión ( $N=36)$.

Tabla 2. Toxicidad debida a bortezomib $(n=36)$.

\begin{tabular}{lcccc}
\hline Toxicidad & Grado 1 & Grado 2 & Grado 3 & Total \\
\hline Polineuropatía & $13(36,1 \%)$ & $3(8,3 \%)$ & $1(2,8 \%)$ & $17(47,2 \%)$ \\
Anemia & $1(2,8 \%)$ & $3(8,3 \%)$ & - & $4(11,1 \%)$ \\
Plaquetopenia & $1(2,8 \%)$ & - & $2(5,6 \%)$ & $3(8,4 \%)$ \\
Neutropenia & - & $1(2,8 \%)$ & $3(8,3 \%)$ & $4(11,1 \%)$ \\
\hline
\end{tabular}

país hasta enero de 2018, la estrategia de tratamiento incluía triple plan con bortezomib, tanto en debut como recaída, particularmente en pacientes con compromiso renal, citogenética adversa y recaída. Desde marzo de 2018, el FNR amplió la cobertura de bortezomib a todos los pacientes al debut y a pacientes en recaída que no hubieran recibido el fármaco en los últimos 12 meses, ni hubieran mostrado refractariedad al mismo.

Inicialmente, bortezomib fue utilizado por vía intravenosa, según la pauta aceptada en esa época. Posteriormente se demostró que el uso subcutáneo no era inferior en resultados, presentando perfil de toxicidad más favorable, en especial por menor toxicidad neurológica. Por este motivo, desde 2013, en nuestro centro se utilizó sistemáticamente por vía subcutánea ${ }^{(14)}$.

En el presente estudio la TRG fue elevada $(79,5 \%)$, superior para pacientes tratados en primera línea, la SG (61\%) con una mediana de seguimiento de 26 meses y la SLP de 35 meses. De los seis pacientes refractarios al fármaco, tres eran de alto riesgo citogenético y cuatro recibieron bortezomib más allá de la primera línea.

El uso de bortezomib asociado a dexametasona para tratamiento de $\mathrm{MM}$ en primera línea mostró RG de $88 \%$, con SG de $87 \%$ a 12 meses ${ }^{(15)}$. Su uso asociado a melfa- lán y prednisona en pacientes no candidatos a trasplante mostró RG de $89 \%$ con $32 \%$ de RC, superiores a melfalán-prednisona, con mayor sobrevida libre de eventos ( $83 \%$ vs $51 \%$ a 16 meses). Además, la citogenética adversa no tuvo impacto en los resultados, sugiriendo efecto beneficioso en esta población de alto riesgo ${ }^{(16)}$.

En el ensayo VISTA, el plan bortezomib-melfalán-prednisona obtuvo mejor tiempo a la progresión (24 meses) y RC (30\%) comparado con melfalán-prednisona, con reducción de $35 \%$ del riesgo de muerte a tres años. La edad y falla renal no afectaron los resultados en los tratados con bortezomib-melfalán y prednisona, y el tiempo a la progresión fue similar en pacientes con alto riesgo citogenético comparado con los pacientes sin alto riesgo $^{(3)}$.

El plan CyBorD mostró RG de 96\%, con 46\% de RC y $71 \%$ de VGPR o superior en pacientes con MM de reciente diagnóstico, siendo la citopenia, hiperglicemia y neuropatía los principales efectos adversos.

$\mathrm{Su}$ uso en pacientes con alto riesgo mostró menor RG que en pacientes sin alto riesgo $(62,5 \%$ vs $86,5 \%)$ en el trabajo de Kropff y colaboradores, similar a lo reportado en otros trabajos, por ejemplo el EVOLUTION trial $^{(17,18)}$. 
En el ensayo fase III del grupo GIMEMA, el plan VTD fue comparado con TD en pacientes candidatos a trasplante y consolidación posterior, siendo VTD superior en términos de $\mathrm{RG}, \mathrm{RC}$ y recaída a tres años. Los pacientes con $\mathrm{t}(4 ; 14)$ mostraron SLP a tres años inferior en pacientes tratados con plan TD, en tanto no hubo diferencia en los tratados con plan VTD. Asimismo, los resultados fueron mejores para el brazo VTD en pacientes con otros factores de riesgo como LDH elevada, del13q, del17p e ISS elevado ${ }^{(11)}$.

En el contexto de recaída, múltiples ensayos avalan su uso en combinación con alquilantes, inmunomoduladores o corticoides ${ }^{(19,20)}$.

En nuestro trabajo la respuesta global fue superior a la reportada internacionalmente en pacientes de alto riesgo tratados con este fármaco. Esto se debe, por una parte, a que la rigurosidad en la asignación del MM como causa de la insuficiencia renal es probablemente inferior a la de los ensayos clínicos, incluyéndose pacientes en los que la insuficiencia renal es de causa multifactorial. La mayoría de los pacientes incluidos en el subgrupo de alto riesgo en nuestro estudio lo fue por insuficiencia renal, siguiendo las recomendaciones del FNR. Sin embargo, el análisis de resultados según estratificación de riesgo en los ensayos referidos, se basa fundamentalmente en el hallazgo de alteraciones citogenéticas, eventualmente incorporando ISS o LDH, sin incluir la insuficiencia renal. Por otra parte, los resultados de los ensayos se analizan según intención de tratamiento, incluyendo pacientes que no completan más de un ciclo, en tanto en nuestro análisis la mediana de tratamientos recibidos fue de seis ciclos, máximo contemplado por la cobertura del FNR.

El uso de bortezomib subcutáneo y semanal se ha asociado a mejor perfil de toxicidad sin detrimento en la eficacia clínica, con reducción de neuropatía ( $14 \%$ vs $34 \%)^{(14)}$.

En nuestro análisis se constató neuropatía emergente en 17 pacientes, siendo solamente un caso grado III. Debemos destacar que 18 pacientes recibieron el tratamiento intravenoso hasta 2013 y no hubo una evaluación por neurólogo al debut en todos los casos. La mitad de los pacientes desarrolló toxicidad neurológica, siendo mayor en los que lo recibieron en forma intravenosa $(55,6 \%$ vs $33 \%)$.

La toxicidad hematológica se reporta frecuentemente con la administración de bortezomib, siendo la plaquetopenia la más frecuente (34\%). En nuestro trabajo la toxicidad hematológica fue inferior a la reportada a nivel internacional. Esto puede explicarse por una diferente pauta de controles paraclínicos en la vida real a los controles realizados en ensayos clínicos de evaluación de fármacos. De todos modos, no hubo complicaciones vinculadas a citopenias que motivaran internación ni fallecimiento.

La incidencia de reactivación de virus herpes zóster es frecuente en pacientes tratados con bortezomib $(14 \%)$, según el ensayo VISTA ${ }^{(3)}$. Por este motivo se recomienda la profilaxis con antivirales sistemáticamente. En nuestro estudio, todos la recibieron y la mayoría no tuvo esta complicación, que fue detectada solo en dos casos.

Los efectos adversos en general fueron leves a moderados y manejables con modificación de dosis. En ningún caso se reportó suspensión del tratamiento por toxicidad severa. El bajo número de pacientes no justifica ampliar el análisis de estos resultados.

La vía intravenosa y subcutánea presentaron eficacia similar, con menor toxicidad neurológica en la administración subcutánea.

Con dificultades derivadas del bajo número de pacientes y la heterogeneidad de presentación y vía de administración del fármaco ya comentadas, los resultados de nuestro análisis se asemejan a lo reportado a nivel internacional, mostrando que bortezomib es un fármaco efectivo y con adecuado perfil de seguridad para el tratamiento del MM de alto riesgo en primera, segunda y tercera línea. Se requiere un mayor número de pacientes para validar los resultados obtenidos.

\section{Resumo}

O mieloma múltiplo é a segunda neoplasia hematológica mais frequente. É uma doença incurável, cuja sobrevida se duplicou nos últimos anos graças aparecimento de novos fármacos. Os esquemas terapêuticos com bortezomibe melhoram a resposta, sobrevida livre de progressão e a sobrevida global. Desde 2009 no Uruguai este fármaco é parcialmente financiado pelo Fondo Nacional de Recursos (FNR). O objetivo deste estudo é analisar a experiência com p uso de bortezomibe em pacientes com diagnóstico de mieloma múltiplo atendidos no Hospital de Clínicas.

Método: estudo observacional, retrospectivo e descritivo; foram incluídos todos os pacientes com diagnóstico de mieloma múltiplo que receberam tratamento com bortezomibe em primeira, segunda e/ou terceira linha no Hospital de Clínicas de Montevidéu Uruguai, de 2009 a 2016.

Resultados: 36 pacientes receberam bortezomibe. O esquema mais utilizado foi ciclofosfamida - bortezomibe - dexametasona. Em 54\% dos pacientes a vida de administração foi subcutânea. A taxa de resposta global foi de 79,5\% (87\% em primeira linha e $68,8 \%$ em segunda ou terceira linha). 43,6\% apresentou polineuropatia e $28,2 \%$ citopenias. Com uma mediana de seguimento de 
26 meses a sobrevida global foi de $61 \%$ e a sobrevida livre de progressão de 35 meses (IC 95\%, 22,6-47,4).

Conclusão: o tratamento com bortezomibe apresentou uma boa taxa de resposta. A neuropatia foi o efeito tóxico mais frequente. Bortezomibe é um fármaco efetivo e com perfil de seguridade adequado para o tratamento do MM em primeira, segunda e terceira linha.

\section{Bibliografía}

1. Rajkumar SV, Dimopoulos MA, Palumbo A, et al. International Myeloma Working Group updated criteria for the diagnosis of multiple myeloma. Lancet Oncol. 2014;15(12): e538-e548. doi: 10.1016/S1470-2045(14)70442-5.

2. Richardson PG, Barlogie B, Berenson J, et al. A Phase 2 Study of Bortezomib in Relapsed, Refractory Myeloma. N Engl J Med. 2003;348(26):2609-2617. doi:10.1056/NEJMoa 030288.

3. Mateos M-V, Richardson PG, Schlag R, et al. Bortezomib Plus Melphalan and Prednisone Compared With Melphalan and Prednisone in Previously Untreated Multiple Myeloma: Updated Follow-Up and Impact of Subsequent Therapy in the Phase III VISTA Trial. J Clin Oncol. 2010;28(13):22592266. doi:10.1200/JCO.2009.26.0638.

4. Kumar SK, Rajkumar SV, Dispenzieri A, et al. Improved survival in multiple myeloma and the impact of novel therapies. Blood. 2008;111(5):2516-2520. doi:10.1182/blood2007-10-116129.

5. Criteria for the classification of monoclonal gammopathies, multiple myeloma and related disorders: a report of the International Myeloma Working Group. $\mathrm{Br} \mathrm{J}$ Haematol. 2003;121(5):749-757.

6. Durie BGM, Harousseau J-L, Miguel JS, et al. International uniform response criteria for multiple myeloma. Leukemia. 2006;20(9):1467-1473. doi:10.1038/sj.leu.2404284.

7. Common Terminology Criteria for Adverse Events (CTCAE) - CTCAE_4.03_2010-06-14_QuickReference_5x7.pdf.

8. Durie BG, Salmon SE. A clinical staging system for multiple myeloma. Correlation of measured myeloma cell mass with presenting clinical features, response to treatment, and survival. Cancer. 1975;36(3):842-854.

9. Greipp PR, San Miguel J, Durie BGM, et al. International staging system for multiple myeloma. J Clin Oncol Off J Am Soc Clin Oncol. 2005;23(15):3412-3420. doi:10.1200/JCO.2005.04.242.

10. Mikhael JR, Dingli D, Roy V, et al. Management of newly diagnosed symptomatic multiple myeloma: updated Mayo Stratification of Myeloma and Risk-Adapted Therapy (mSMART) consensus guidelines 2013. Mayo Clin Proc. 2013;88(4):360-376. doi:10.1016/j.mayocp.2013.01.019.
11. Cavo M, Tacchetti P, Patriarca F, et al. Bortezomib with thalidomide plus dexamethasone compared with thalidomide plus dexamethasone as induction therapy before, and consolidation therapy after, double autologous stem-cell transplantation in newly diagnosed multiple myeloma: a randomised phase 3 . Lancet (London, England). 2010;376(9758):2075-2085. doi: 10.1016/S0140-6736(10)61424-9.

12. Barlogie B, Anaissie $\mathbf{E}$, van Rhee $\mathbf{F}$, et al. Incorporating bortezomib into upfront treatment for multiple myeloma: early results of total therapy 3. Br J Haematol. 2007;138(2):176-185 doi:10.1111/j.1365-2141.2007.06639.x.

13. Richardson PG, Sonneveld P, Schuster MW, et al. Safety and efficacy of bortezomib in high-risk and elderly patients with relapsed multiple myeloma. Br J Haematol. 2007;137(5):429-435. doi:10.1111/j.1365-2141.2007.06585.x.

14. Moreau P, Pylypenko H, Grosicki S, et al. Subcutaneous versus intravenous bortezomib in patients with relapsed multiple myeloma: subanalysis of patients with renal impairment in the phase III MMY-3021 study. Haematologica. 2015;100(5):e207-210. doi:10.3324/haematol.2014.118182.

15. Jagannath S, Durie BGM, Wolf J, et al. Bortezomib therapy alone and in combination with dexamethasone for previously untreated symptomatic multiple myeloma. $\mathrm{Br} \mathrm{J}$ Haematol. 2005;129(6):776-783. doi:10.1111/j.1365-2141.2005.05540.x.

16. Mateos M-V, Hernández J-M, Hernández M-T, et al. Bortezomib plus melphalan and prednisone in elderly untreated patients with multiple myeloma: results of a multicenter phase 1/2 study. Blood. 2006;108(7):2165-2172. doi:10.1182/ blood-2006-04-019778.

17. Kropff M, Liebisch P, Knop S, et al. DSMM XI study: dose definition for intravenous cyclophosphamide in combination with bortezomib/dexamethasone for remission induction in patients with newly diagnosed myeloma. Ann Hematol. 2009;88(11):1125-1130. doi:10.1007/s00277-009-0726-6.

18. Kumar S, Flinn I, Richardson PG, et al. Randomized, multicenter, phase 2 study (EVOLUTION) of combinations of bortezomib, dexamethasone, cyclophosphamide, and lenalidomide in previously untreated multiple myeloma. Blood. 2012;119(19): 4375-4382. doi:10.1182/blood-2011-11-395749.

19. Mele G, Giannotta A, Pinna S, et al. Frail elderly patients with relapsed-refractory multiple myeloma: efficacy and toxicity profile of the combination of bortezomib, high-dose dexamethasone, and low-dose oral cyclophosphamide. Leuk Lymphoma. 2010;51(5):937-940. doi:10.3109/10428191 003695660.

20. Ahn J-S, Yang D-H, Jung S-H, et al. A comparison of bortezomib, cyclophosphamide, and dexamethasone (Vel-CD) chemotherapy without and with thalidomide (Vel-CTD) for the treatment of relapsed or refractory multiple myeloma Ann Hematol. 2012;91(7):1023-1030. doi:10.1007/s00277. 012-1420-7. 